\title{
The interaction of pronunciation rules and lexical representations in reading aloud
}

\author{
MARY BETH ROSSON \\ Thomas J. Watson Research Center, IBM Corporation, Yorktown Heights, New York
}

\begin{abstract}
Two experiments examined the role of pronunciation rules and of lexical information in pronouncing letter strings. In Experiment 1, subjects pronounced pseudowords varying in the strength of the rules needed to pronounce them, as well as in the availability of a lexical model. In Experiment 2 , the stimuli were words varying in rule strength and in usage frequency. The pronunciation times from both experiments displayed an interaction between rules and lexical information: When the rules necessary were strong, the relative availability of lexical information was less important than when the rules were weak. The results were discussed with respect to both traditional dual-process models of pronunciation and models proposing the use of lexical analogies.
\end{abstract}

A salient feature of skilled reading is the rapid generation of pronunciations, both of known words and of novel but pronounceable nonwords, or pseudowords. Currently, a debate exists concerning the appropriate underlying model for these pronunciation processes. A major piece of the controversy concerns the unit of analysis in pronunciation. A traditional view (e.g., Coltheart, 1978; Coltheart, Davelaar, Jonasson, \& Besner, 1977; Forster \& Chambers, 1973) has been that readers possess pronunciation knowledge of two types: lexical representations that contain pronunciations for known words, and an independent set of pronunciation rules (typically presumed to be grapheme-phoneme correspondences) used to pronounce unfamiliar words and pseudowords. The current debate concerns the existence of grapheme-based pronunciation rules, with a variety of researchers suggesting that lexical information may be sufficient to support the pronunciation of both known and novel strings.

\section{Are Pronunciation Rules Necessary?}

The fact that readers easily generate pronunciations for completely novel words has often been taken as prima facie evidence for the existence and use of pronunciation rules. Typically, readers' reliance on such rules has been incorporated into a dual-process model (Baron \& Strawson, 1976; Forster \& Chambers, 1973) in which readers look up lexical items and apply pronunciation rules in parallel, with the former generally faster for known items, but the latter required for novel or infrequent words. Recently, however, researchers have begun to question this view of pronunciation.

This research was submitted to the University of Texas at Austin in partial fulfillment of the requirements for a doctorate in psychology. Thanks go to Tom Carr, two anonymous reviewers, and the editor for constructive comments on earlier versions of the paper. Requests for reprints should be sent to Mary Beth Rosson, IBM Watson Research Center, P.O. Box 218, Yorktown Heights, NY 10598.
One particularly damaging piece of evidence involves the contribution of lexical information to the pronunciation of novel words or pseudowords. The proposal has been that a novel string might be pronounced by analogy to a known word that is visually similar (Baron, 1977; Brooks, 1977; Glushko, 1979; Marcel, 1980, Rosson, 1983). So, for example, Kay and Marcel (1981) found that the pronunciation chosen for an ambiguous pseudoword (a nonword that might be pronounced in more than one way, such as VEAD) could be manipulated by preceding it with a known word containing the string in question (preceding VEAD with DEAD increases frequency of the /ved/ pronunciation). This finding, although certainly of interest, might be due to the priming of alternate spelling-sound correspondences rather than to pronunciation by analogy to lexical entries. That is, perhaps in the process of pronouncing DEAD, readers momentarily strengthen an association between EA and $\mid \varepsilon /$, thus increasing the likelihood that it will influence subsequent pronunciation of VEAD (Kay \& Marcel, 1981, p. 405, discussed this possibility). However, Rosson (1982) reported the converging finding that the pronunciation of an ambiguous pseudoword can be biased indirectly, by preceding it with a word semantically related to a lexical entry. In other words, simply increasing the activation of a useful known word affects the pronunciation chosen for the novel word (e.g., preceding VEAD with ALIVE increases the likelihood that it will be pronounced /ved/ to rhyme with DEAD).

These findings provide good evidence that specific lexical sites can contribute to the pronunciation of words never encountered before. As a result, one of the major arguments for positing an independent set of graphemebased pronunciation niles has been weakened. Note, however, that although it may well be true that lexical information guides the pronunciation of nonwords, we know very little about how this might actually take place. The "analogy" mechanism captures only the notion of a partial visual match between a nonword and a lexical item- 
retrieving the IVE in BIVE from GIVE, for example. We have no idea how the reader comes by the remaining information (here, the starting sound) or how these various pieces are combined to yield a full pronunciation. Even this example oversimplifies matters immensely, since there is also the problem of suppressing competing contributions (e.g., the IVE in DIVE). Nonetheless, these data do constrain the form of possible pronunciation models, in that they must be such as to allow for lexical effects on nonword pronunciation.

Additional problems for proponents of dual-process models arise from recent research on the regularity effect. In the past, regular words (those that follow the "rules" of English, such as HATE) have been observed to enjoy a pronunciation advantage over exception words (those that break the rules, such as HAVE) (Baron \& Strawson, 1976; Gough \& Cosky, 1977; Stanovich \& Bauer, 1978). This finding has been attributed to the simultaneous occurrence of the lexical look-up and rule application processes, with the faster of the two "winning" on any given occasion. Exception words are at a disadvantage because they have only one potentially successful path to pronunciation--lexical look-up-whereas regular words have two.

Glushko (1979) provided an alternative account of the phenomenon. He argued that the regularity effect has been confounded with a consistency effect, in that regular words are simply those most likely to bear letter patterns with a consistent pronunciation across words. Thus, for example, FEET is a regular word; it also possesses only consistent lexical "neighbors" (for Glushko, words possessing the same ending, such as BEET, MEET). In contrast, not only is BEEN an exception word, but it also possesses lexical neighbors with conflicting pronunciations (e.g., SEEN, TEEN). Glushko pointed to the existence of "inconsistent regular" words such as SEEN, whose pronunciations follow the rules, but which possess lexical neighbors with competing phonological representations. He reported that consistent regular words such as FEET are pronounced more quickly than their inconsistent counterparts, and that this advantage appears for consistent over inconsistent pseudowords as well (e.g., REET vs. REEN). Glushko argued that the standard distinction between lexical look-up and rule application provides no mechanism for the interfering contributions from visually similar lexical items. He offered an activationsynthesis model of pronunciation, in which any visual string-word or nonword-activates a number of lexical forms that are then synthesized to produce an appropriate pronunciation. The inconsistency effect appears because the presence of competing phonological correspondences makes the synthesis more difficult.

Although Glushko's (1979) results are intriguing, there may be a problem with their generality. Seidenberg and Tanenhaus (1982) reported a series of studies suggesting that much of the inconsistency effect observed by Glushko (1979) may have been due to the design of his study, in which subjects were asked to pronounce conflicting lexi- cal neighbors within the same block of stimuli, thereby promoting an interference effect. Seidenberg and Tanenhaus found an interaction between the presence of conflicting lexical neighbors and the frequency of the target word: For high-frequency words, the presence of competing lexical entries had no effect, but an inconsistency effect did appear for low-frequency words.

A third source of evidence problematic for the dualprocess view comes from studies of persons with reading dysfunction. Thus, for example, patients with surface dyslexia make oral reading errors related primarily to the spelling-sound characteristics of the stimulus, and their comprehension appears to be based on the pronunciations they generate. This behavior is consistent with a model positing separate rule and lexical mechanisms-the argument is that the lexical route is not available to these readers, but that the rule mechanism still functions moderately well. Marcel (1980) questioned this account, however, as a consequence of his own analyses of patients' reading errors. In his analyses, lexical information does influence the errors that are made: He has observed correspondences between the syntactic and frequency classes of the correct and error responses, and many of the "spelling-related" errors appear to bear some lexical relation to the presented word. Like Glushko (1979), Marcel proposed that all strings, both words and nonwords, are pronounced with reference to lexical representations.

\section{The Interaction of Rules and Lexical Knowledge}

We have discussed a number of recent findings that are problematic for theories proposing separate contributions by pronunciation rules and lexical representations. A major problem comes from demonstrations that lexical information can contribute to the pronunciation of novel words as well as known words, because this severely weakens the claim that a separate body of rule knowledge is needed to account for new pronunciations. Furthermore, it appears that the regularity effect observed for known words may be due more to the lack of competing correspondences than to an advantage accrued by simultaneous contributions from pronunciation rules.

One response to such findings is simply to dismiss the notion of a rule-based pronunciation process. That is, given the questionable status of an independent set of rules, then a pronunciation process involving both lexical look-up and rule application no longer seems feasible. This is the direction taken by researchers such as Glushko $(1979,1981)$ and Marcel $(1980$; Kay \& Marcel, 1981). They propose instead a unified representation of pronunciation knowledge, in which all correspondences are stored together and are operated upon by the same mechanism.

Although this approach seems to be an economical solution to the new evidence accruing, a number of issues remain unresolved. As mentioned earlier, there is as yet no mechanism worked out for how one actually synthesizes a pronunciation for a novel word in such a system. 
In addition, we do not know the nature of the spelling units activated. In particular, the status of letter-based analysis is quite unclear. Given the emphasis on lexical information in the work described thus far, one might imagine that word-level analysis is primary in pronunciation, with information about smaller units available only as the result of secondary activation or as the result of computations performed on the word pronunciations. If so, then lexical factors (e.g., the frequency of a word, the presence of conflicting lexical neighbors, the availability of a lexical model in pseudoword pronunciation) should dominate the pronunciation process. However, if activation of letter-based units occurs simultaneously, then we should observe effects produced by this level of analysis as well.

This latter suggestion embodies a rather different way of thinking about pronunciation rules. Instead of the traditional notion of a rule, in which a single graphemephoneme correspondence is given "regular" status (e.g., ee $\rightarrow / \mathrm{i} /$ ), we might now think of rules as reflecting all grapheme-phoneme correspondences. No longer would there be a single sound correspondence for a given grapheme unit. Indeed, any relationship observed for more than one word would be a candidate "rule"; the distinctions between this knowledge and knowledge about word pronunciations would be the size of the unit and the fact that the correspondence has been observed over multiple word types. Such a conceptualization bears some resemblance to the representation described by Rumelhart and McClelland (1980) in their parallel-activation model of word processing. A model like this offers great flexibility, in that simultaneous excitatory and inhibitory connections among word and letter representations can provide a rich set of contributions to be used in accounting for particular findings. But this degree of flexibility is also a potential weakness. An important step will be the establishment of constraints on the patterns of activation allowed by such models.

The present work was designed to provide an empirical contrast between the use of knowledge at the word versus letter level in pronunciation. In both experiments, one of pseudoword pronunciation and the other of word pronunciation, the activation of word-based lexical information and that of letter-based rule information were pitted against one another. This was accomplished by manipulating variables expected to influence the time course of activation at the two levels.

The manipulation of rule activation was based on an important assumption: A given rule was assumed to vary in strength as a function of its type frequency, the number of English words it appears in. Type frequency (rather than token frequency) was chosen for two reasons. First, it better reflects the classic view of rule or category formation, in which a feature's predictive value is a function of the number of exemplars that express it (e.g., Rosch, Simpson, \& Miller, 1976). Second, empirical data exist that suggest that it is the number of different words a correspondence appears in, not its cumulative usage frequency, that predicts pronunciation of an ambiguous seg- ment in a pseudoword (Kay \& Marcel, 1981, p. 407). A corollary to this rule-strength assumption, of course, is that rules of lower type frequency will be applied more slowly, because their weaker representations will result in less activation during the pronunciation process.

The availability of lexical knowledge was manipulated in a more traditional fashion. For pseudowords, the manipulation consisted of simply the presence or absence of a relevant lexical model; for words, the manipulation was of the word's usage frequency. Here, the assumption was that, for pseudowords with close lexical neighbors (and words of higher frequency), information about the pronunciation of word units would be more quickly available than that about pseudowords with no neighbors (or words of low frequency). Consequently, pseudowords with neighbors and words with higher frequency should be at an advantage in the lexically driven process.

If the lexical and rule activation occur in parallel, an interaction should obtain between the rule-strength and lexical-availability variables. When rule strength is high, a pronunciation should be quickly generated from rule application, so that the availability of lexical information should be less important than when the necessary rules are weak and slow to apply.

\section{EXPERIMENT 1}

Consider the two pseudowords DORCE and DOICE. Each has a single lexical neighbor of relatively higher frequency (FORCE and VOICE; "lexical neighbor" is defined here as a word that differs from the pseudoword by a single letter). Given a model in which pronunciation of such pseudowords is driven largely by the activation of lexical neighbors (e.g., Glushko, 1981), and assuming that the items are equated for orthographic and articulatory factors, the two items should be pronounced with approximately equal ease. However, the strings do differ in another potentially important respect: All of the component spelling-sound rules in DORCE have relatively high type frequencies (i.e., each appears in many different English words), whereas DOICE includes one rule $(\mathrm{OI} \rightarrow / \mathrm{oy} /)$ that has a relatively low type frequency.

In the experiment, pseudowords were created to reflect the crossing of two levels of rule strength (high or low frequency) and two levels of lexical availability (neighbor present or absent), whereas other variables expected to affect naming times (e.g., orthographic structure, initial voiced sounds) were held constant. It was expected that when rule strength was low, the presence of a lexical neighbor would facilitate pronunciation, but that when rule strength was high, the availability of lexical information such as this would have little or no effect.

\section{Method}

\section{Subjects}

The participants were 32 undergraduates from an undergraduate psychology class; they participated in partial fulfillment of a course requirement. All subjects had normal or corrected-to-normal sight and hearing. A second group of 20 served as subjects in a control 
condition designed to investigate possible articulatory differences among the experimental conditions.

\section{Stimuli}

Experimental variables. Stimuli were generated from a base set of words selected from the Carroll, Davies, and Richman (1971) corpus. The base words were all of high to moderate frequency, were four or five letters in length, represented "regular' " spellingsound correspondences (following the rules of Venezky, 1970), and contained unique cores (spellings following their initial consonants). Each base word was first scored for the strength of the spellingsound rules it represented. This was accomplished by breaking each word into its constituent rules (according to Venezky, 1970) and determining the number of English words exemplifying each rule (according to the counts of Hanna, Hanna, Hodges, \& Rudorf, 1966). The score assigned to a given word was the logarithm of the type frequency of its weakest rule. A weakest-rule score was used, rather than an average, because pilot work (Rosson, 1982, p. 30) had suggested that the former was strongly related to naming time, whereas the latter was not; logarithms were used to compensate for the skewed distribution of rule frequencies.

The base words were then converted into pseudowords that represented the crossing of two factors, rule strength and the presence or absence of a lexical neighbor. The initial consonant of each base word was replaced to create a pseudoword; this was done for one group of base words whose weakest rules were of relatively high frequency (e.g., FORCE was transformed to $\mathrm{KORCE}$; the weakest rule is $\mathrm{C}(\mathrm{E}) \rightarrow / \mathrm{s} /$, with a log frequency of 2.52) and another group whose weakest rules were of low frequency (e.g., VOICE became LOICE; the weakest rule is $\mathrm{OI} \rightarrow$ loy/, with a $\log$ frequency of 0.90 ). These two groups represented the strongrule and weak-rule neighbor-present conditions. The two neighborabsent conditions were created by generating, for each item in the first two sets, a string possessing the same minimum-frequency rule, but that was at least two letters removed from any lexical neighbor (defined here as a known word differing only in its initial consonant). To finish the example, MURCE was paired with KORCE and MOITE with LOICE.

Control variables. During creation of the stimuli, three control variables were also monitored. One of these was the usage frequency of the base word; because the two sets of neighbor-present pseudowords were created from different base words, it was important to ensure that the frequencies (and, hence the lexical availability) of the neighbors were equivalent. The average Standard Frequency Index (SFI; Carroll, Davies, \& Richman, 1971) was 53.32 for the weak-rule neighbor-present condition and 53.87 for the strong-rule neighbor-present stimuli.

The orthographic structure of the experimental stimuli was also monitored. Because the stimuli were presented visually, it was necessary to control for variables likely to affect visual processing, and many researchers (see Carr, Posner, Pollatsek, \& Snyder, 1979, and Massaro, Venezky, \& Taylor, 1979) have argued that orthographic structure is one such variable. Although orthographic regularity was ensured by making all of the items legal English strings, additional control was imposed by equating conditions for average log bigram frequency (based on the counts of Mayzner \& Tresselt, 1965). The average values were $2.53,2.51,2.54$, and 2.52 for the strong-rule neighbor-present, strong-rule neighborabsent, weak-rule neighbor-present, and weak-rule neighbor-absent conditions, respectively.

The final variable controlled was the voicing of initial consonants: This was done in an attempt to remove gross articulation-onset differences among the sets of pseudowords. Each of the four groups contained nine items with voiced initial consonants and six without.

In sum, four sets of pseudowords were generated, representing the crossing of two variables and the control of three others. The items in two of the sets possessed lexical neighbors but varied in the strength of their weakest rules (e.g., KORCE has strong rules; LOICE contains a weak rule); the other two sets had no neighbors and varied in the same way with respect to rule strength (e.g. MURCE vs. MOITE). Given the stringent controls imposed, the number of acceptable stimuli was small-there were 15 items in each group. The 60 pseudowords were inserted in random order into a list of 60 filler words, so that the final pronunciation list contained half words and half pseudowords. An additional 16 items, half of which were words and half pseudowords similar to the experimental items, were appended to the beginning of the list and served as practice items.

\section{Procedure}

The subjects were tested individually in a small, dimly lit room containing a CRT and a voice-key apparatus. They were told they would be presented with 136 words on the screen, 1 at a time, and that their task was to pronounce each one as quickly and as accurately as they could. They were warned that some of the words were "made up for the experiment, " but that they should simply pronounce them as well as they could, as though the "made-up" words were new words seen for the first time. They were instructed that because there would be only about $1 \mathrm{sec}$ between words, they should try to refrain from correcting any errors they noticed.

Presentation of the stimuli was controlled by a PDP-8I computer and began 1,000-1,500 msec after the subject first depressed a footpedal to indicate readiness to begin. Each stimulus remained on the screen until the subject's vocal response, which triggered a voice-activated relay by means of a throat microphone worn snugly around the neck. There was a variable intertrial interval $(1,000$ $1,500 \mathrm{msec}$ ) between items in the list. If a subject removed his/her foot from the pedal, a break in list presentation was instantiated; the subjects were told of this feature and were encouraged to take a break at any time if they felt their attention was lagging. However, most subjects chose to work through the list continuously, a task requiring only about 4 or $5 \mathrm{~min}$.

The PDP-8I recorded articulation onset latencies for each string. In addition, the experimenter monitored the subjects' pronunciations via an intercom, transcribing pronunciations of all experimental stimuli for subsequent review and scoring.

Naming control. An independent group of subjects pronounced the experimental items under different conditions. These subjects viewed each pseudoword on the CRT screen until they had decided how it should be pronounced. At that point, they pressed a button, and after a variable interval (1,000-1,500 msec), a prompt ("*”) appeared on the screen. The task was to produce the preassigned pronunciation as quickly as possible upon appearance of the prompt. The subjects were instructed not to "anticipate" the pronunciation by preparing their mouth shape; in order to discourage this, they were asked to keep the mouth in a neutral position (with the tip of the tongue inserted between lips) between vocal responses. The time from appearance of the prompt to onset of the pronunciation response was recorded by the computer, and the pronunciation was transcribed by the experimenter for scoring.

\section{Results}

The subjects' mean correct pronunciation latencies served as the dependent measures in a $2 \times 2$ analysis of variance, with both rule strength (strong vs. weak) and neighbor availability (present vs. absent) as within-subject factors. In calculating these means, reaction times greater than 2.5 standard deviations from a subject's grand mean were replaced by that criterion value. The latencies for incorrect pronunciations were discarded. A pronunciation was counted as an error if it included a spelling-sound correspondence not represented in at least one English word. The group means for each condition are presented in Table 1, along with the error rates. 
Table 1

Mean Group Pronunciation Latencies (in Milliseconds) and Percent Error for Experiment 1

\begin{tabular}{|c|c|c|c|c|}
\hline & \multicolumn{2}{|c|}{ Neighbor Present } & \multicolumn{2}{|c|}{ Neighbor Absent } \\
\hline & Mean & $\mathrm{PE}$ & Mean & $\mathrm{PE}$ \\
\hline & \multicolumn{2}{|c|}{ KORCE } & \multicolumn{2}{|c|}{ MURCE } \\
\hline \multirow[t]{2}{*}{ Strong Rules } & 651 & 1.9 & 650 & 5.2 \\
\hline & \multicolumn{2}{|c|}{ DOICE } & \multicolumn{2}{|c|}{ MOITE } \\
\hline Weak Rules & 675 & 8.1 & 716 & 13.1 \\
\hline
\end{tabular}

Note $-P E=$ percent error.

The analysis of variance revealed significant main effects of both rule strength $[F(1,31)=28.04, p<.001]$ and neighbor availability $[\mathrm{F}(1,31)=13.31, \mathrm{p}<.001]$. In general, pronunciation times were faster for pseudowords composed of strong rules; they were also faster for nonwords having close lexical neighbors. However, interpretation of these main effects must be tempered in light of the strong interaction between the two factors $[F(1,31)$ $=23.11, \mathrm{p}<.001]$. Examination of the means in Table 1 suggests that the interaction is due to the absence of a neighbor effect for strong-rule items. Tests of simple main effects confirmed this suggestion: The presence of weak rules increased times for nonwords with neighbors $[F(1,31)=14.96, p<.001]$ as well as for those without neighbors $[\mathrm{F}(1,31)=113.79, \mathrm{p}<.001]$. However, although the availability of lexical neighbors facilitated pronunciation of weak-rule items $[F(1,31)=42.29, p<$ $.001]$, it had no effect on strong-rule items $(F<1)$.

A parallel analysis of variance was conducted on the subjects' mean numbers of errors in each condition. This analysis revealed significant effects of both rule strength $[\mathrm{F}(1,31)=25.82, \mathrm{p}<.001]$ and neighbor availability $[F(1,31)=15.50, p<.001]$. The subjects made more errors on words containing weak rules and on words without lexical models. However, in contrast to the analysis of reaction times, the error data reflected no interaction between the two factors $(F<1)$.

\section{Naming Control}

The subjects' mean correct naming control times were calculated using the same criterion for outliers and errors described for the analysis of pronunciation times. Group means for the four conditions are presented in Table 2, along with error rates. The analysis of variance (again,

Table 2

Mean Group Naming Control Latencies (in Milliseconds) and Percent Error for Experiment 1

\begin{tabular}{|c|c|c|c|c|}
\hline & \multicolumn{2}{|c|}{ Neighbor Present } & \multicolumn{2}{|c|}{ Neighbor Absent } \\
\hline & Mean & $\mathrm{PE}$ & Mean & PE \\
\hline & & & & \\
\hline \multirow[t]{2}{*}{ Strong Rules } & 393 & 1.7 & 393 & 1.7 \\
\hline & \multicolumn{2}{|c|}{ DOICE } & \multicolumn{2}{|c|}{ MOITE } \\
\hline Weak Rules & 393 & 3.7 & 399 & 8.0 \\
\hline
\end{tabular}

Note-PE = percent error. with both factors within subjects) revealed no effects of rule strength or neighbor availability and no interaction between the two variables (all Fs $<1$ ); there were no systematic articulation differences between the four groups of items. The analysis of errors made in this control task, however, revealed effects similar to those observed for the original pronunciation task: The subjects made more errors on weak-rule items $[F(1,19)=14.11, p<.001]$, although the facilitative effect of neighbor was only marginal $[F(1,19)=3.60, p<.08]$. There was also a marginal interaction of the two variables $[\mathrm{F}(1,19)=3.31$, $\mathrm{p}<.09]$. These data suggest that the manipulations of rule strength and neighbor availability were strong enough to affect accuracy even when the subjects were allowed as much time as they wished to make their pronunciation decisions.

\section{Discussion}

As expected, both rule strength and neighbor availability produced strong effects on readers' pronunciation performance. The expectation that these sources of information would interact was also supported: When the rules necessary to pronounce a word were strong, there appeared to be no facilitative effect of a lexical model. In contrast, when the rules needed were weak, the existence of a useful lexical model aided pronunciation considerably.

These findings are easily accounted for by a model proposing simultaneous activation of lexical and rule knowledge. In such a model, two types of activation would take place after a string is presented and converted into some visual representation. The letters and letter groups in the string would combine to produce activation of one or more lexical entries and at the same time would produce direct activation of grapheme-phoneme correspondences. Because the strings would be novel, none of the lexical items would match completely, and therefore the pronunciation information available from these sites would need some type of modification (e.g., the stripping off of one or more phonemes) to offer usable information. Thus, the speed with which a lexical item contributed useful information would depend not only on the degree to which it is activated by the visual stimulus, but also on the complexity of any modifications that must be performed. Meanwhile, rule activation would be taking place, with its time course a function of the strength of the graphemephoneme correspondences activated.

Of the items pronounced by subjects in this experiment, half were assumed to provide a fair amount of activation of at least one lexical entry, namely, the word differing in only initial consonant. The other half were assumed to produce no strong activation of any lexical item. Thus, fully half of the strings were in a position to receive contributions from word pronunciations. But because the activation of lexical knowledge was assumed to occur in parallel with that of rules, the observable effects of these contributions were expected to depend on the time course of the concurrent rule activation. Half of the items able 
to receive contributions from the lexicon were designed to be pronounceable by strong, unambiguous rules; the other half were pronounceable by weak rules exemplified in only a few English words. For the former, the rule knowledge should have been rapidly available, often yielding a phonological representation quickly enough to make irrelevant any information being prepared by the lexically driven process. In contrast, for strings associated with the more difficult rules, the phonological conversion via rule application would have been delayed sufficiently for effects of lexical contributions to be observed.

The details of the interaction observed between rule strength and neighbor availability are also of interest. Specifically, the fact that there was no effect of neighbor availability for items composed of easy rules, whereas the effect of rule strength was present even for items with lexical neighbors, points to the primacy of the grapheme-phoneme rules for this set of stimuli. That is, in the case of the strong-rule items, rule activation appeared fast enough to "beat" the lexical input on virtually every occasion, resulting in no observable effect of neighbor availability. Furthermore, the availability of a neighbor for weak-rule items was not sufficient to reduce their latencies to a level comparable to that of either group of strong-rule items. Of course, how much this particular pattern of results is due to the level of the two independent variables implemented for the experiment is unclear; if the differences between strong and weak rules had been less, and those between levels of neighbor availability greater, a different pattern might well have appeared.

Another complicating factor for this interpretation lies in the error rates observed. Recall that in the error data, main effects of both rule strength and neighbor availability were present, but there was no interaction of the two factors. That is, in these data, the facilitative effect of a potential lexical model was independent of the strength of the rules they embodied. These findings raise the possibility that some degree of speed-accuracy trade-off was taking place in the strong-rule, neighbor-absent condition, relative to the strong-rule, neighbor-present condition. Perhaps the familiarity of the constituent rules in the former group provoked a quick response before the full phonological code was available, causing a reduction in latency at the expense of accuracy. It may be that if subjects had delayed their responses to these items long enough to ensure accuracy, an effect of neighbor availability would have obtained for the strong-rule items as well as for the weak-rule ones.

\section{EXPERIMENT 2}

Experiment 1 provided evidence about the relative contributions of lexical and rule information to the pronunciation of pseudowords. But the model providing the context for the work is intended as a general model of pronunciation. applicable to both novel and familiar words. That is, it assumes that both rule-based and lexical routes are available during the pronunciation of known words as well as that of pseudowords. Of course, in the case of known words, the lexical mechanism is expected to operate much more rapidly than it does for pseudowords. This is a consequence of two factors: A complete visual match would be expected to produce more rapid and intense activation of a stored lexical item; and the phonological information available at a lexical site needs no operation performed on it-it already represents the complete pronunciation desired. Given that rule and lexical activation occur simultaneously, the lexical route is more likely to finish first for words than for pseudowords. Nevertheless, it may still be possible to find evidence of rule contributions to word pronunciation, particularly when the word in question is of low frequency.

Experiment 2 manipulated the same two factors crossed in Experiment 1. lexical availability and rule strength. However, in this case, the lexical variable was manipulated by varying the frequency of the naming target itself, rather than the presence or absence of a lexical neighbor. As for the nonword naming task, an interaction between the two variables was expected, with rule strength producing a much larger effect for words of low frequency.

\section{Method}

\section{Subjects}

The participants were 32 undergraduates from an introductory psychology class. Their service was in partial fulfillment of a course requirement. All were native English speakers and had normal or corrected-to-normal sight and hearing. A separate group of 18 subjects served under the naming control condition.

\section{Stimuli}

As in Experiment 1, four- and five-letter monosyllabic English words with unique cores (spellings following the initial consonant) served as the initial pool of items. Each item was scored for its $\log$ minimum-rule frequency and its SFI. Four sets of items, representing the crossing of rule strength and word frequency, were chosen as follows. First, items with moderate to high SFIs whose minimum rules were either frequent (e.g., DEPTH, whose weakest rule is $\mathrm{TH} \rightarrow / \theta /$ ) or infrequent (e.g., SOLVE, whose weakest rule is $\mathrm{O}-\mathrm{E} \rightarrow / \mathrm{a} /$ ) were selected. Each of these items was then paired with a word possessing a low SFI, but bearing the same minimumfrequency rule (BERTH and COPSE, respectively). One item in the strong-rule condition did not have an acceptable low-frequency counterpart with the identical weak rule. so a word containing a different rule of similar frequency was chosen instead.

The final set of items was determined by simultaneously equating the groups on several dimensions. Due to the matching used in selecting the two groups of strong- and weak-rule words. the levels of rule strength were constant across levels of word frequency, with the two weak-rule groups having average log minimurn-rule frequencies of 1.55 and 1.55 and the two strong-rule groups having averages of 2.65 and 2.64 . Similarly, groups were equated for word frequency across levels of rule strength. Because of other constraints, perfect equivalence was not possible: Average SFI for the weakrule. high-frequency condition was slightly lower than that for the strong-rule. high-frequency condition (53.4 vs. 56.4). However. the values for the low-frequency conditions differed in a similar fashion. with averages of 35.4 for the weak-rule set and 38.8 for the strong-rule set. In other words, the frequency differences within levels of nule strength were almost identical. Because the interaction of the lactors was of most interest. these frequency values were 
judged to be acceptable. Finally, groups were equated for articulatory and orthographic variables, such that the four groups had equal numbers of voiced versus unvoiced initial consonants (seven of each in all groups) and equivalent average log bigram frequencies (2.73, $2.73,2.72$, and 2.72 for the weak-rule, high- and low-frequency, and strong-rule, high- and low-frequency conditions, respectively).

In sum, four sets of words were selected that represented the orthogonal crossing of rule strength and word frequency, with bigram frequency and voicing controlled across groups. Because of the severe constraints imposed, only 14 acceptable items in each condition could be found. The 56 experimental items were inserted in random order into a list of 56 filler words, with 18 practice words appended to the beginning of the list.

\section{Procedure}

The procedure was identical to that described for Experiment 1, except that no mention of nonwords was made, because none occurred in this pronunciation list. The subjects averaged about $4 \mathrm{~min}$ to complete the 130 -word list. A separate group of undergraduates named only the 56 experimental words under the naming control conditions described for Experiment 1.

\section{Results}

The subjects' mean correct naming latencies for each condition of the $2 \times 2$ design served as dependent measures in a two-way analysis of variance, with both rule strength and word frequency serving as within-subjects factors. Because these were word stimuli, the detection of errors was fairly straightforward; any response not corresponding to a word's actual pronunciation was discarded. As in Experiment 1, reaction times of more than 2.5 standard deviations from a subject's grand mean were replaced with that criterion value. Condition means with errors in parentheses appear in Table 3.

The analysis of variance revealed significant main effects of both rule difficulty $[F(1,31)=41.48, p<.0001]$ and word frequency $[\mathrm{F}(1,31)=42.95, \mathrm{p}<.0001]$. The subjects were faster at pronouncing words composed of strong rules; they were also faster for the higher frequency words. As in Experiment 1, however, the main result was an interaction between the two factors $[F(1,31)=22.23$, $\mathrm{p}<.001$ ]. In fact, examination of the means suggests that both main effects are due entirely to the difficulty of the weak-rule, low-frequency words. Tests of simple main effects confirmed this suspicion: Neither the effect of rule strength on high-frequency words nor the effect of word frequency on strong-rule words was significant $[F(1,31)=0.00, F(1,31)=1.44$, respectively $]$. In con-

Table 3

Mean Group Pronunciation Latencies (in Milliseconds) and Percent Error for Experiment 2

\begin{tabular}{|c|c|c|c|c|}
\hline & \multicolumn{2}{|c|}{ High-Frequency Word } & \multicolumn{2}{|c|}{ Low-Frequency Word } \\
\hline & Mean & PE & Mean & PE \\
\hline & & & & \\
\hline \multirow[t]{2}{*}{ Strong Rules } & 583 & 1.1 & 594 & 2.4 \\
\hline & \multicolumn{2}{|c|}{ SOLVE } & \multicolumn{2}{|c|}{ COPSE } \\
\hline Weak Rules & 583 & 0.6 & 654 & 8.2 \\
\hline
\end{tabular}

Note-PE = percent error.
Table 4

Mean Group Naming Control Latencies (in Milliseconds) and Percent Error for Experiment 2

\begin{tabular}{|c|c|c|c|c|}
\hline & \multicolumn{2}{|c|}{ High-Frequency Word } & \multicolumn{2}{|c|}{ Low-Frequency Word } \\
\hline & Mean & $\mathrm{PE}$ & Mean & PE \\
\hline & & & & \\
\hline \multirow[t]{2}{*}{ Strong Rules } & 395 & 1.6 & 381 & 1.2 \\
\hline & \multicolumn{2}{|c|}{ SOLVE } & \multicolumn{2}{|c|}{ COPSE } \\
\hline Weak Rules & 394 & 1.2 & 390 & 4.0 \\
\hline
\end{tabular}

Note $-P E=$ percent error.

trast, both comparisons involving the weak-rule, lowfrequency condition were significant $[F(1,31)=44.24$, $p<.0001$, for the effect of rule strength on lowfrequency words; $F(1,31)=61.92, p<.0001$, for the effect of word frequency on weak-rule words].

A parallel analysis on the subjects' mean numbers of errors in each condition yielded the same pattern of results, with more errors being produced for weak-rule words $[F(1,31)=9.62, p<.005]$ and for low-frequency words $[F(1,31)=33.70, p<.0001]$. As in the latency analysis, the interaction of the two factors was also significant $[F(1,31)=12.76, p<.002]$, with the greatest number of errors in the weak-rule, low-frequency condition.

\section{Naming Control}

The subjects' mean correct naming latencies in the naming control procedure are presented in Table 4, along with the error rates in each condition. The data underwent the same analysis described for the pronunciationlatency data. Neither of the independent variables produced a main effect $[F<1$ for the effect of rule strength, and $F(1,17)=2.50, p>.10$, for word frequency]. The interaction of the two factors also failed to reach significance $[F(1,17)=2.36, \mathrm{p}>.10]$.

\section{Discussion}

These results suggest that the manipulation of rule and lexical activation affect word pronunciation in ways similar to those observed for pseudoword pronunciation in $\mathrm{Ex}$ periment 1. Just as Experiment 1 found that pseudoword naming latencies were influenced by neighbor availability and rule strength, Experiment 2 found that latencies for words were a function of both their frequencies and the strength of the rules they embodied. Furthermore, the finding of an interaction between the variables in Experiment 2 suggests that, as was the case for pseudoword naming, the two types of activation occur in parallel.

The form of the interaction observed for words differs from that observed for pseudowords. Recall that, for pseudowords, there appeared to be no lexical contributions to the pronunciation of strong-rule stimuli, although the effect of rule strength was present for pseudowords with and without lexical neighbors. In contrast, the word latencies reflect no effect of rule strength for high- 
frequency words, and, although the difference was not significant, there was a tendency for the frequency effect to appear for strong-rule words as well as for weak-rule ones. (This tendency would be magnified if the articulation data from the control experiment were taken into account, because they imply that the mean for the highfrequency, strong-rule items may have been lowered somewhat by ease of articulation; see Table 4.) An admittedly liberal interpretation of this pattern suggests that, for these word stimuli, lexical information almost always "beats" the rules for high-frequency words, regardless of the difficulty of the rules embodied in the item. Rule strength influences reaction times only when word frequency is low.

\section{GENERAL DISCUSSION}

The experiments reported here combine to provide support for a view of pronunciation driven both by lexical associations between words and their pronunciations, and by grapheme-phoneme correspondence rules whose strength varies with the number of words exemplifying them. Furthermore, these two sources of knowledge contribute in parallel, as evidenced by the interactions observed. When the relevant rule knowledge is strong, the availability of specific lexical information becomes less critical; when the rules are weak, however, specific lexical knowledge contributes significantly to pronunciation. This interaction between rule and lexical knowledge obtains for both words and pseudowords, with the form of the interaction in each case easily accounted for by the amount of activation expected at specific lexical sites.

The results of Experiment 1 also extend the findings of lexical contributions to the pronunciation of pseudowords. In the past, the evidence for this has come from the pronunciation of "ambiguous" pseudowords, nonwords such as VEAD that have competing lexical models. Although the finding of Rosson (1983) suggests that lexical sites can indeed be involved in the pronunciation of this class of pseudowords, it was not clear that similar effects would be observed in the pronunciation of "consistent" pseudowords. The finding of Experiment 1-facilitative effects of lexical neighbors for strings like DOICE that have only one, regular neighbor-suggests that a more general contribution of lexical information to pseudoword pronunciation does occur.

Although Experiment 1 took an important step toward generalizing the involvement of lexical items in the pronunciation of novel strings, the process needs much more analysis. Thus, for example, the studies described and reported here studied pseudowords that varied from their lexical models in a very specific way-through substitution of the initial consonant or consonant cluster. Additional research (Rosson, 1982) suggests that similar effects on pronunciation can be found for pseudowords modified in the internal vowel or final consonant, but the relationship between known and novel strings must be studied in much more depth.
A similar caveat holds for the notion of "rule" espoused here. Clearly, the work made a number of assumptions about what constitutes a rule and about the variables expected to influence rule activation. Thus, for example, although the manipulation of rule strength as a function of word-type frequency had dramatic effects on pronunciation latencies, it is very difficult to separate this type frequency measure from one based on token frequencies. Because normative data on token frequencies were not available, it was impossible to contrast the two measures. A related concern involves the potential contributions of rule consistency. Very often, rules with few exemplars have related rules that may compete during pronunciation. For instance, $\operatorname{SC}(E) \rightarrow / s /$ is a low-frequency correspondence; although it may be a less effective rule for this reason alone, there is also a related rule $\mathrm{SC} \rightarrow / \mathrm{sk} /$ that quite possibly competes with it during pronunciation of a word such as SCENT. More research will be necessary to work out the structural variables controlling the activation of subword units during pronunciation.

The experiments reported here have been motivated and discussed in the context of parallel-activation models of pronunciation. It is important to note, however, that modifications could be made to dual-process models that would allow them to account for these results as well. Thus, for example, if one allowed a rule mechanism that included rules varying in strength, as proposed here, and one allowed a lexical look-up mechanism that could provide lexical input to the pronunciation of visually similar words, then the modified model would make the same predictions as the parallel-activation scheme described here. Indeed, not only do the current data not distinguish between these two general classes of models, but also there is some doubt as to whether such models can be empirically distinguished at all (see Carr \& Pollatsek, in press, for a discussion of these problems). If not, we may be forced to rely on other considerations, such as parsimony (Anderson, 1978) in choosing between them.

The role that these data do play is the provision of empirical constraints for models of pronunciation. Thus, a strong version of the dual-process model, in which rules offer a single regular correspondence for any graphemic unit, and in which lexical information contributes only to the pronunciation of known words, can be rejected. Modified versions of dual-process models will be forced to include mechanisms providing for word-level contributions to nonword pronunciation, as well as for the interaction between the two levels of analysis. Similarly, strong versions of an analogy model, which assume that all strings are pronounced with reference to specific lexical items, can be rejected. Instead, such models will have to provide appropriate strength parameters for word-to-letter links to produce both the word-level and letter-level effects observed here. A potentially important constraint lies in the argument that the strength of rule knowledge is determined differently from that of lexical information, with one based on type frequency and the other on token frequency. If this assumption is verified by future re- 
search, it will have a large impact on models of pronunciation, because it implies that the representation of grapheme-phoneme correspondences differs from lexical entries in a qualitative fashion. However, this issue is still an open one, because the current data are only consistent with a type-frequency measure and do not provide evidence against a token-frequency measure.

In sum, although the current work does not allow us to distinguish between all variants of dual-process versus parallel-activation models, it does argue against some of the stronger forms of these models, and it does provide an important set of results that must be explained by future models. In particular, it has shown that both wordlevel and letter-level knowledge contribute to the pronunciation of words and nonwords. And it has shown that these two sources of knowledge interact in this process, such that when one source of information is strong, the effect of the other is considerably diminished.

\section{REFERENCES}

ANDERSON, J. R. (1978). Arguments concerning representations for mental imagery. Psychological Review, 85, 249-277.

BARON, J. (1977). Mechanisms for pronouncing printed words: Use and acquisition. In D. LaBerge \& S. Samuels (Eds.), Basic processes in reading: Perception and comprehension. Hillsdale, NJ: Erlbaum.

Baron, J., \& Strawson, C. (1976). Use of orthographic and wordspecific knowledge in reading words aloud. Joumal of Experimental Psychology: Human Perception and Performance, 2, 386-393.

BrooKs, L. (1977). Non-analytic correspondences and pattern in word pronunciation. In J. Requin (Ed.), Attention and performance VII. Hillsdale, NJ: Erlbaum.

CARr, T. H., \& PollatSEK, A. (in press). Recognizing printed words: A look at current models. In D. Besner, T. G. Waller, \& G. E. MacKinnon (Eds.), Reading research: Advances in theory and practice (Vol. 5). New York: Academic Press.

Carr, T. H., Posner, M. I., Pollatsek, A., \& Snyder, C. R. R. (1979). Orthography and familiarity effects in word processing. Journal of Experimental Psychology: General, 108, 389-414.

Carroll, J. B., Davies, B., \& Richman, B. (1971). The American Heritage word frequency book. New York: American Heritage.

Coltheart, M. (1978). Lexical access in simple reading tasks. In G. Underwood (Ed.), Strategies of information processing. London: Academic Press.
Coltheart, M., Davelaar, E., Janasson, J. T., \& Besner, D. (1977). Access to the internal lexicon. In S. Dornic (Ed.), Attention and performance $V I$. Hillsdale, NJ: Erlbaum.

Forster, K. I., \& Chambers, S. M. (1973). Lexical access and naming time. Journal of Verbal Learning and Verbal Behavior, 12, 627-635.

GLUSHKo, R. J. (1979). The organization and activation of orthographic knowledge in reading aloud. Journal of Experimental Psychology: Human Perception and Performance, 5, 674-691.

GlushKo, R. J. (1981). Principles for pronouncing print: The psychology of phonography. In A. M. Lesgold \& C. A. Perfetti (Eds.), Interactive processes in reading. Hillsdale, NJ: Erlbaum.

Gough, P. B., \& Cosky, M. J. 1977). One second of reading again. In N. J. Castellan, D. B. Pisoni, \& G. R. Potts (Eds.), Cognitive theory (Vol. 2). Hillsdale, NJ: Erlbaum.

Hanna, P. R., Hanna, J. S., Hodges, R. E., \& Rudorf, E. H., JR. (1966). Phoneme-grapheme correspondences as cues to spelling improvement (HEW Rep. No. OE-32008). Washington, DC: Department of Health, Education, and Welfare.

KaY, J., \& MarCEL, A. J. (1981). One process, not two, in reading aloud: Lexical analogies do the work of non-lexical rules. Quarterly Journal of Experimental Psychology, 33, 397-413.

MarCEL, A. J. (1980). Surface dyslexia and beginning reading: A re vised hypothesis of the pronunciation of print and its impairments. In M. Coltheart, K. Patterson, \& J. C. Marshall (Eds.), Deep dyslexia. London: Routledge and Kegan Paul.

Massaro, D. W., Venezky, R. L., \& TAYlor, G. A. (1979). Orthographic regularity, positional frequency, and visual processing of letter strings. Journal of Experimental Psychology: General, 108, 107-124.

Mayzner, M. S., \& Tresselt, M. E. (1965). Tables of single-letter and digram frequency counts for various word-length and letter position combinations. Psychonomic Monograph Supplements, 1, 13-32.

Rosch, E., Simpson, C., \& Miller, R. S. (1976). Structural bases of typicality effects, Journal of Experimental Psychology: Human Perception and Performance, 2, 491-502.

Rosson, M. B. (1982). Lexical and rule contributions to word and pseudoword pronunciation. Unpublished doctoral dissertation, University of Texas, Austin.

Rosson, M. B. (1983). From SOFA to LOUCH: Lexical contributions to pseudoword pronunciation. Memory \& Cognition, 11, 152-160.

Rumelhart, D. E., \& MCClelland, J. L. (1980). An interactive activation model of the effect of context in perception: Part II (CHIP Rep. No. 95). San Diego: University of California, Center for Human Information Processing.

Seidengerg, M. S., \& Tanenhaus, M. K. (1982, November). Spellingsound correspondences and reading. Paper presented at the meeting of The Psychonomic Society. Minneapolis.

Stanovich, K. E., \& Bauer, D. W. (1978). Experiments on the spelling-to-sound regularity in word recognition. Memory \& Cognition, 6, 410-415

VENEZKY, R. L. (1970). The structure of English orthography. The Hague: Mouton.

\section{APPENDIX}

Item Means for Experiments 1 and 2

Item Means for Experiment 1

\begin{tabular}{|c|c|c|c|c|c|c|c|c|c|c|c|}
\hline \multicolumn{6}{|c|}{ Strong Rules } & \multicolumn{6}{|c|}{ Weak Rules } \\
\hline \multicolumn{3}{|c|}{ Neighbor } & \multicolumn{3}{|c|}{ No Neighbor } & \multicolumn{3}{|c|}{ Neighbor } & \multicolumn{3}{|c|}{ No Neighbor } \\
\hline Item & RT & $\mathrm{PE}$ & Item & RT & $\mathrm{PE}$ & Item & $\mathrm{RT}$ & $\mathrm{PE}$ & Item & $\mathrm{RT}$ & $\mathrm{PE}$ \\
\hline kenth & 740 & 6.3 & dunth & 607 & 6.3 & loice & 655 & 3.1 & moite & 648 & 18.8 \\
\hline esk & 596 & 0.0 & henk & 581 & 6.3 & virl & 600 & 0.0 & lirn & 613 & 9.4 \\
\hline vurst & 652 & 0.0 & mursh & 650 & 18.8 & hoap & 685 & 0.0 & doag & 689 & 3.2 \\
\hline hanch & 720 & 3.2 & kanth & 670 & 3.2 & hirst & 633 & 6.3 & kirnt & 772 & 9.4 \\
\hline repth & 639 & 0.0 & ripth & 658 & 0.0 & mype & 664 & 15.6 & myre & 653 & 21.9 \\
\hline dilm & 631 & 0.0 & vilp & 656 & 9.4 & kamb & 646 & 6.3 & hemb & 664 & 0.0 \\
\hline geeth & 628 & 0.0 & geesh & 662 & 6.3 & koubt & 866 & 12.5 & hoibt & 905 & 40.6 \\
\hline lurb & 640 & 0.0 & nurm & 623 & 3.2 & hulse & 598 & 6.4 & rulte & 722 & 6.4 \\
\hline nich & 662 & 9.4 & hech & 695 & 9.4 & gauce & 795 & 21.9 & rauve & 702 & 9.4 \\
\hline
\end{tabular}




$\begin{array}{lrlllllllllr}\text { nulf } & 637 & 0.0 & \text { vulm } & 587 & 0.0 & \text { nazz } & 597 & 6.4 & \text { nozz } & 638 & 18.9 \\ \text { korce } & 678 & 3.2 & \text { murce } & 621 & 0.0 & \text { nuit } & 643 & 21.9 & \text { guid } & 797 & 37.5 \\ \text { hidth } & 635 & 0.0 & \text { ledth } & 629 & 9.4 & \text { molve } & 634 & 0.0 & \text { nolse } & 630 & 6.3 \\ \text { hund } & 687 & 0.0 & \text { kuld } & 723 & 3.2 & \text { kuice } & 797 & 12.5 & \text { huine } & 997 & 3.2 \\ \text { melf } & 575 & 0.0 & \text { nilf } & 611 & 9.4 & \text { doard } & 704 & 0.0 & \text { voart } & 660 & 3.2 \\ \text { vurve } & 631 & 6.3 & \text { hurke } & 757 & 0.0 & \text { vamn } & 647 & 12.5 & \text { vomn } & 742 & 18.8\end{array}$

Item Means for Experiment 2

\begin{tabular}{|c|c|c|c|c|c|c|c|c|c|c|c|}
\hline \multicolumn{6}{|c|}{ Strong Rules } & \multicolumn{6}{|c|}{ Weak Rules } \\
\hline \multicolumn{3}{|c|}{ High Frequency } & \multicolumn{3}{|c|}{ Low Frequency } & \multicolumn{3}{|c|}{ High Frequency } & \multicolumn{3}{|c|}{ Low Frequency } \\
\hline Item & RT & PE & Item & RT & $\mathrm{PE}$ & Item & $\mathrm{RT}$ & PE & Item & RT & $\mathrm{PE}$ \\
\hline fifth & 592 & 0.0 & filth & 647 & 9.4 & solve & 602 & 3.2 & copse & 880 & 31.2 \\
\hline depth & 583 & 0.0 & berth & 568 & 0.0 & keith & 658 & 0.0 & sheik & 675 & 15.6 \\
\hline ranch & 561 & 0.0 & perch & 555 & 0.0 & scene & 606 & 0.0 & scent & 631 & 0.0 \\
\hline teeth & 599 & 0.0 & tenth & 567 & 0.0 & quest & 653 & 3.2 & quill & 688 & 3.2 \\
\hline curve & 577 & 0.0 & slurp & 628 & 0.0 & wheel & 560 & 0.0 & whine & 579 & 6.4 \\
\hline force & 605 & 0.0 & horde & 634 & 3.2 & guard & 602 & 0.0 & guile & 722 & 18.8 \\
\hline speak & 666 & 0.0 & leash & 534 & 3.2 & rhode & 593 & 0.0 & rhine & 631 & 0.0 \\
\hline midst & 558 & 3.2 & tempt & 605 & 0.0 & ghost & 560 & 0.0 & ghoul & 708 & 25.0 \\
\hline bench & 545 & 0.0 & conch & 784 & 3.2 & write & 573 & 0.0 & wrest & 658 & 0.0 \\
\hline width & 589 & 3.2 & lurch & 548 & 3.2 & court & 599 & 0.0 & mourn & 618 & 3.2 \\
\hline burst & 560 & 0.0 & burnt & 586 & 0.0 & knite & 505 & 0.0 & knoll & 625 & 3.4 \\
\hline huge & 610 & 3.2 & dupe & 598 & 3.2 & type & 548 & 3.2 & tyke & 672 & 3.2 \\
\hline size & 606 & 12.5 & doze & 550 & 0.0 & soap & 556 & 0.0 & $\operatorname{coax}$ & 661 & 12.5 \\
\hline desk & 515 & 0.0 & mesh & 538 & 3.2 & jazz & 556 & 0.0 & fizz & 611 & 0.0 \\
\hline
\end{tabular}

Note $-R T=$ reaction time; $P E=$ percent error.

(Manuscript received January 30, 1984;

revision accepted for publication November 26,1984 .)

\section{ANNOUNCEMENT}

\section{Change in Editorship of Memory \& Cognition}

Robert Bjork completed his regular 4-year term as editor of Memory \& Cognition in 1985. The Publications Committee of the Governing Board of The Psychonomic Society is pleased to announce that Alice F. Healy has agreed to be the new editor (1986-1989). After January 1, 1985, please send manuscripts to Dr. Healy at: Department of Psychology, University of Colorado, Muenzinger Building, Campus Box 345, Boulder, CO 80309. 